\title{
Inhibitory effect and mechanism of exogenous mammalian sterile 20-like kinase 1 on the growth of human colorectal cancer
}

\author{
JIAN WU ${ }^{1,2^{*}}$, XIAOHONG YANG ${ }^{1 *}$, HONGFEI LU ${ }^{1}$, LIQIAO LIU $^{1}$, BAOHUA XU $^{3}$, \\ SHUANGYAN ZHENG ${ }^{3}$, BO YU ${ }^{1}$, KEMIN JIE $^{1}$ and FUSHENG WAN ${ }^{1}$ \\ ${ }^{1}$ Department of Biochemistry and Molecular Biology, Basic Medical College of Nanchang University, Nanchang, \\ Jiangxi 330006; ${ }^{2}$ Department of Medical Technology, Jiangxi Medical College, Shangrao, Jiangxi 334003; \\ ${ }^{3}$ Experimental Animal Center, Nanchang University, Nanchang, Jiangxi 330006, P.R. China
}

Received August 5, 2015; Accepted November 3, 2016

DOI: $10.3892 / 01.2017 .5786$

\begin{abstract}
The present study aimed to observe the inhibitory effect and preliminary mechanism of exogenous mammalian sterile 20-like kinase 1 (MST1) on the growth of colorectal cancer SW480 cells. The SW480 cells were randomly divided into the following groups: Control, empty enhanced green fluorescent protein (EGFP) plasmid (pEGFP-N1), MST1 EGFP plasmid (pEGFP-MST1), $20 \mu \mathrm{mol} / 1$ fluorouracil (5-FU) and pEGFP-MST1 + 5-FU. An MTS colorimetric assay was used to detect cell viability, Hoechst 33342 staining was used to observe cell apoptosis, and western blotting and immunohistochemistry were used todetect the levels of the proteins MST1,yes-associated protein (YAP), phospho-YAP1 (Ser127), p53 and p53 upregulated modulator of apoptosis (PUMA). In addition, nude mice were injected with SW480 cells to assess the tumor inhibition rates. Compared with the control group, the growth inhibition and apoptosis rates, the levels of MST1, p53 and PUMA, and the ratios of phospho-YAP1/YAP in the pEGFP-MST1 and pEGFP-MST1 + 5-FU groups were increased significantly $(\mathrm{P}<0.01)$. Additionally, relative to the control group, the tumor inhibition rates in the nude mice transplanted with SW480 cells of the pEGFP-MST1 and pEGFP-MST1 + 5-FU groups were $48.52 \pm 1.63$ and $87.28 \pm 2.58 \%$, respectively, and the positive rates of phospho-YAP1 (Ser127) protein in nuclei increased significantly $(\mathrm{P}<0.01)$. Overall, exogenous MST1 effectively inhibited the proliferation and growth of transplanted human colorectal cancer cells and promoted cancer cell apoptosis. The
\end{abstract}

Correspondence to: Dr Fusheng Wan, Department of Biochemistry and Molecular Biology, Basic Medical College of Nanchang University, 461 Bayi Avenue, Nanchang, Jiangxi 330006, P.R. China

E-mail:wanfs01@163.com; fushengwandoc@126.com

*Contributed equally

Key words: human mammalian sterile 20-like kinase 1, colorectal cancer, apoptosis, yes-associated protein, gene therapy mechanism involved may be associated with the increase of intracellular phospho-YAP1 (Ser127) protein.

\section{Introduction}

Colorectal cancer is one of the most common types of malignant tumor in humans, ranking third in the world with respect to incidence (1). The incidence and mortality of colorectal cancer in China is increasing, and the mortality rate has risen to the fourth highest of all types of malignant tumor (2). It has been confirmed that the occurrence and development of colorectal cancer is closely associated with the abnormal activity of the Hippo signaling pathway (3-5). The Hippo signaling pathway is a highly conserved growth control signaling pathway that was identified in Drosophila in 1995 (6), for which mammalian sterile 20-like kinase 1 (MST1) is one of the core members. MST1 and MST2 are members of the class II germinal center kinase family, with $76 \%$ of their amino acid sequence in common (7). In 2005, Huang et al (8) first identified the signaling transduction pathway of the Hippo pathway from the cytoplasm to cell nuclei in Drosophila, thus regulating the expression of downstream genes, cell growth, proliferation and apoptosis. Dong et al (9) subsequently established the yes-associated protein (YAP) conditional transgenic mouse model, and determined the signal transduction orders of the MST1-YAP pathway in mammals. Zhou et al (10) found that mice lacking MST1/2 could not inhibit the proliferative and anti-apoptotic activity of the carcinogenic gene YAP1, resulting in the formation of liver cell carcinoma. However, subsequent to the transfection of MST1 into HepG2 cells, MST1 was re-expressed, resulting in the loss of the proliferative activity of YAP1, the promotion of apoptosis and the elimination of tumorigenicity (11).

The effect of exogenous MST1 on preventing and treating colorectal cancer has not yet been reported. With the development of molecular targeting therapy, numerous tumor-associated signaling molecules have become therapeutic targets, and targeted therapy may become a novel direction in the treatment of colorectal cancer. The present study transfected the MST1 gene into SW480 cells, aiming to investigate the inhibitory effect and mechanism of exogenous MST1 on the growth of human colorectal cancer SW480 cells grafted 
in nude mice, and to provide novel experimental evidence for targeted therapy against colorectal cancer.

\section{Materials and methods}

Transfection. PolyJet ${ }^{\mathrm{TM}}$ in vitro DNA transfection reagent (cat. no. SL100688; SignaGen Laboratories, Rockville, MD, USA) was used to mediate the transfection of the MST1 recombinant enhanced green fluorescent protein (EGFP) plasmid pEGFP-N1-MST1 and empty pEGFP-N1 plasmids (Clontech Laboratories, Inc., Mountainview, CA, USA) into human colorectal cancer SW480 cells (purchased from Cell Library Of Typical Culture Collection Committee, Chinese Academy Of Sciences). Firstly, SW480 cells were cultured in high-glucose (H)-Dulbecco's modified Eagle's medium (DMEM) complete culture medium (Solarbio, Beijing, China) plus $10 \%$ fetal calf serum (TransGen Biotech, Beijing, China), $100 \mathrm{U} / \mathrm{ml}$ penicillin, and $100 \mathrm{mg} / \mathrm{ml}$ streptomycin (Gibco; Thermo Fisher Scientific, Inc., Waltham, MA, USA) in 6-well plates until $60-70 \%$ of the culture area was covered. The original medium was then discarded, $900 \mathrm{ml}$ fresh complete medium was added into each well, and the cells were incubated at $37^{\circ} \mathrm{C}$ for 30-60 min. Transfection solution $(50 \mu \mathrm{l})$ was added to each well, which was then gently mixed and cultured at $37^{\circ} \mathrm{C}$ and $5 \% \mathrm{CO}_{2}$ for $5 \mathrm{~h}$. The transfection solution was subsequently replaced with DMEM containing $10 \%$ fetal bovine serum and the cells were re-cultured at $37^{\circ} \mathrm{C}$ for $24-48 \mathrm{~h}$. The transfection efficiency was observed and images were captured with a fluorescence microscope.

The transfection solution was prepared as follows: Solution A, $1 \mathrm{~g}$ plasmid was dissolved in $50 \mu 1$ serum-free DMEM culture and pipetted 3-4 times; solution B, $3 \mu$ l PolyJet reagent solution was added to $50 \mu \mathrm{l}$ serum-free DMEM and pipetted 3-4 times; and solution C (transfection solution), solution B was quickly added to solution A, rapidly pipetted 3-4 times and left to stand at room temperature for $15 \mathrm{~min}$.

Experimental grouping. The colorectal cancer SW480 cell line were cultured in H-DMEM complete culture medium containing $100 \mathrm{U} / \mathrm{ml}$ penicillin and $100 \mathrm{U} / \mathrm{ml}$ streptomycin at $37^{\circ} \mathrm{C}$ and $5 \% \mathrm{CO}_{2}$ for $48-78 \mathrm{~h}$. Cells in the logarithmic growth phase were then randomly divided into 5 groups: i) Control group, subsequent to seeding the cells into the plate, the culture medium was discarded the next day and replaced by serum-free culture medium for a 6-h culture, then the culture medium was discarded and replaced by complete medium for another 48-h culture; ii) the pEGFP-N1 group, transfected with the empty pEGFP-N1 plasmid and cultured for $48 \mathrm{~h}$; iii) the pEGFP-MST1 group, transfected with the pEGFP-N1-MST1 plasmid and cultured for $48 \mathrm{~h}$; iv) the fluorouracil (5-FU) group, $20 \mu \mathrm{mol} / 1$ 5-FU (Ameresco Inc., Framingham, MA, USA) was added 1 day after seeding, then cultured for $48 \mathrm{~h}$; and v) the pEGFP-MST1 + 5-FU group, transfected by the pEGFP-MST1 plasmid on the next day of seeding and cultured for $24 \mathrm{~h}$, prior to adding $20 \mu \mathrm{mol} / 15$-FU for another $24-\mathrm{h}$ culture.

MTS colorimetric assay. The cells were harvested and a single cell suspension was prepared with H-DMEM, at a density of $1 \times 10^{3}-1 \times 10^{4}$ cells/well. The cells were seeded into 96-well plates, with $200 \mu \mathrm{l}$ in each well and the edge well filled with sterile phosphate-buffered saline (PBS). The cells which were divided into 5 groups and cultured at $37^{\circ} \mathrm{C}$ and $5 \% \mathrm{CO}_{2}$ for 24,48 or 72 h. A total of $20 \mu 1$ MTS (Sigma-Aldrich; EMD Millipore, Billerica, MA, USA) was then added to each well, and the culture was terminated subsequent to $4 \mathrm{~h}$ and agitated for $10 \mathrm{~min}$ to fully dissolve the crystals. The absorbance of each well was measured at $490 \mathrm{~nm}$ and the inhibition rate was calculated. Cell growth inhibition rate $(\%)=1$-cell survival rate $\mathrm{OR}=(1$-absorbance of the experimental group at $490 \mathrm{~nm} /$ absorbance of the control group at $490 \mathrm{~nm}) \times 100$.

Hoechst 33342 staining. The SW480 cells were collected and seeded into 12 -well plates at a density of $1 \times 10^{5}$ cells/well; the cell concentrations were adjusted so that the adherent cells reached $70-90 \%$ of the culture area when transfected. The 12 -well plates were cultured at $37^{\circ} \mathrm{C}$ and $5 \% \mathrm{CO}_{2}$ for $48 \mathrm{~h}$, then the culture medium was discarded and the plates were washed with PBS 2-3 times. A total of $500 \mu$ l Hoechst 33342 (Beyotime Institute of Biotechnology, Haimen, China) dye/well was added in darkness and cultured at $37^{\circ} \mathrm{C}$ and $5 \%$ $\mathrm{CO}_{2}$ for 20-30 min. The Hoechst 33342 dye was then discarded and the plates were washed with PBS 2-3 times. The level of apoptosis was observed and images were captured under a fluorescence microscope, with the number of total and apoptotic cells counted in 10 randomly selected high-power fields to calculate the cell apoptotic rate. Apoptotic rate=apoptotic cells/total cells $\mathrm{x} 100$.

Western blot analysis. SW480 cells were washed with PBS and lysed with radioimmunoprecipitation assay (RIPA) cell lysis reagent containing proteinase and phosphatase inhibitors (Solarbio) at $4^{\circ} \mathrm{C}$ for $30 \mathrm{~min}$. Cell extracts were centrifuged at $12,000 \times \mathrm{g}$ for $20 \mathrm{~min}$ at $4^{\circ} \mathrm{C}$, and the supernatants containing total proteins were mixed with an equal volume of $5 \mathrm{X}$ sodium dodecyl sulfate (SDS) loading buffer. Samples were heated at $95^{\circ} \mathrm{C}$ for $5 \mathrm{~min}$, separated by $12 \%$ gel electrophoresis, and then electrotransferred onto $0.22-\mathrm{mm}$ polyvinylidene difluoride membrane. The membranes were then incubated with antibodies (dilution, 1:200) against p53-upregulated modulator of apoptosis (PUMA; cat. no. 55120-1-AP; ProteinTech Group, Inc., Chicago, IL, USA), p53 (cat. no. 2524S; Cell Signaling Technology, Inc., Danvers, MA, USA), MST1 (cat. no. ab51134; Abcam, Cambridge, UK), YAP1 (cat. no. 4912; Cell Signaling Technology, Inc.) and phospho-YAP (cat. no. ab76252; Abcam) overnight at $4^{\circ} \mathrm{C}$. The membranes were then cultured with horseradish peroxidase-conjugated goat anti-rabbit (cat. no. ZDR-5306) and goat anti-mouse (cat. no. ZDR-5307) secondary antibodies (ZSGB-BIO, Beijing, China; dilution, $1: 2,000)$ for $1.5 \mathrm{~h}$. An anti- $\beta$-actin monoclonal antibody (cat. no. SC-47778; Santa Cruz Biotechnology, Inc., Dallas, TX, USA; dilution, 1:1,000) was used as an internal control. The protein signals were detected by the enhanced chemiluminescence (ECL) detection system (Pierce; Thermo Fisher Scientific, Inc.). The gray scale of the protein bands was scanned using Image-Pro Plus 6.0 (Media Cybernetics, Inc., Rockville, MD, USA). The intensity of the western blot bands was quantified using Image J 1.4.3.67 Launcher Symmetry software (National Institutes of Health, Bethesda, MD, USA). The relative protein level was calculated as follows: Relative protein level $=0.25 \mathrm{x}$ (gray scale ratio between target gene 

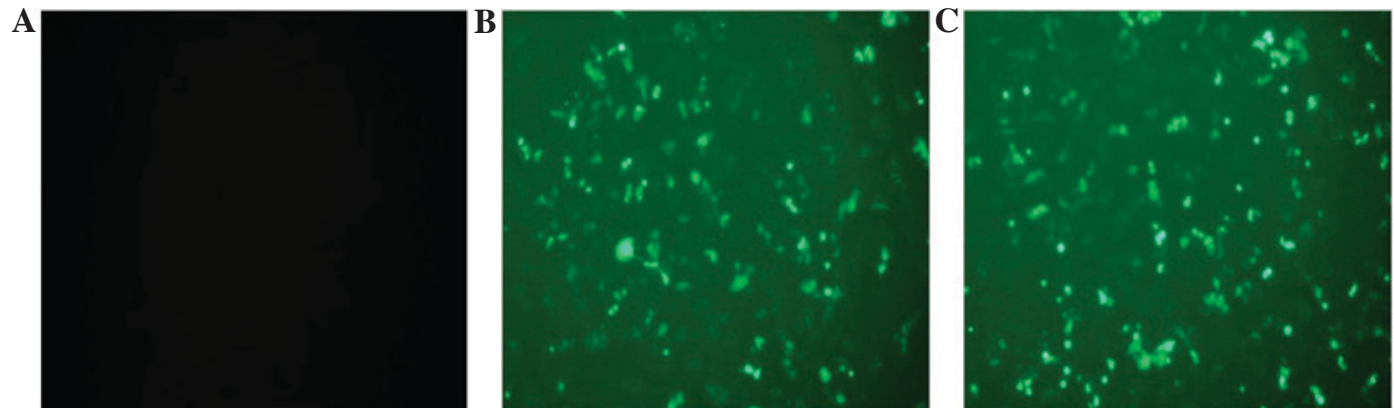

Figure 1. Transfection efficiency of SW480 cells (magnification, x100). (A) Control cells; (B) cells transfected with empty pEGFP-N1; (C) cells transfected with pEGFP-MST1. pEGFP, enhanced green fluorescent protein plasmid.

product bands and $\beta$-actin protein bands in the experimental group)/(gray scale ratio between target gene product bands and $\beta$-actin protein bands in the control group).

Animal experiments. A total of $40 \mathrm{BALB} / \mathrm{c}$ mice (specific pathogen-free, 4-week-old, female mice, weighing $20 \mathrm{~g}$ and purchased from Hunan SJA Laboratory Animal Co., Ltd., Changsha, China) were raised in a sterile environment in laminar flow cabinets with disinfectant-treated baskets and bedding, adequate feed and drinking water, aseptic operation, constant temperature $\left(18-22^{\circ} \mathrm{C}\right)$, constant humidity (50-60\%). SW480 cells $\left(1 \times 10^{6}\right)$ were injected subcutaneously into both the left and right sides of the backs of the mice. When the diameter of the tumor mass reached 2-3 $\mathrm{mm}$, the mice were randomly divided into 5 groups: The control group, in which mice were administered $0.2 \mathrm{ml}$ saline via multi-point injections in tumor and peri-tumoral areas; the negative control group (pEGFP-N1), in which mice were administered a mixture of $0.2 \mathrm{ml}$ pEGFP-N1 plasmid $(100 \mu \mathrm{g})$ and PolyJet in vitro DNA transfection reagent (1:2) via multi-point injections in tumor and peri-tumoral areas; the experimental group (pEGFP-MST1), in which mice were administered a mixture of $0.2 \mathrm{ml}$ pEGFP-MST1 plasmid $(100 \mu \mathrm{g})$ and liposomes (1:2) via multi-point injections in tumor and peri-tumoral areas; the positive control group (5-FU), in which mice were administered $0.2 \mathrm{ml}$ saline-configured 5-FU at a dose of $10 \mathrm{mg} / \mathrm{kg}$ body weight of nude mice, intraperitoneally injected; and the combination therapy group (pEGFP-MST1 + 5-FU), in which mice were intraperitoneally injected with $0.2 \mathrm{ml} 5$-FU $(10 \mathrm{mg} / \mathrm{kg})$ and administered, via multi-point injection, the mixture of $0.2 \mathrm{ml}$ pEGFP-MST1 plasmid $(100 \mu \mathrm{g})$ and liposomes (1:2) in tumor and peri-tumoral areas simultaneously. Each group was administered treatment once every 3 days, for a total of 21 days. On day 27, the mice were sacrificed by cervical dislocation, images were captured and tumor weights were measured to calculate the tumor inhibition rate using the following formulae: Tumor volume $\left(\mathrm{cm}^{3}\right)=0.5 \mathrm{x}$ long diameter $\mathrm{x}$ short diameter ${ }^{2}$; tumor inhibition rate $(\%)=($ mean tumor weight of the control group-mean tumor weight of the treatment group)/mean tumor weight of the control group x100. The present study was performed in strict accordance with the recommendations in the Guide for the Care and Use of Laboratory Animals of the National Institutes of Health. The animal use protocol was reviewed and approved by the Institutional Animal Care and Use Committee of Nanchang University (Nanchang, China).
Immunohistochemistry. Subsequent to measuring the tumor volumes, the mice were sacrificed and the tumor tissues were removed and flushed with saline to remove the blood. The tumor tissues were then weighed and images were captured. The tumors were cut into $3-\mu \mathrm{m}$ sections with scissors, fixed with $4 \%$ paraformaldehyde overnight and embedded in paraffin. The tissues were then dewaxed, and the expression levels of MST1, phospho-YAP1 (Ser127) and PUMA proteins were detected by immunohistochemistry, according to the protocol of the immunohistochemistry kit (cat. no. PV-9000; ZSGB-BIO, Beijing, China). The appearance of deep yellow to dark-brown particles inside the nuclei or cytoplasm was considered as a positive result.

Statistical analysis. SPSS 19.0 (IBM SPSS, Armonk, NY, USA) statistical software was used to analyze the experimental data. The parameters amongst the groups were analyzed with a one-way analysis of variance or unpaired Student's t-test and the pairwise comparison among groups was analyzed using Dixon's q test. $\mathrm{P}<0.05$ was considered to indicate a statistically significant difference.

\section{Results}

Transfection efficiency of plasmids. As shown in Fig. 1, cells transfected with pEGFP-N1 and pEGFP-MST1 plasmids exhibited green fluorescence under the fluorescence microscope, while the control group exhibited no fluorescence, suggesting that the plasmids containing pEGFP-N1 or pEGFP-MST1 had entered the SW480 cells.

Effect of overexpression of MST1 on colorectal cancer SW480 cell proliferation. Fig. 2 shows that compared with the control or pEGFP-N1 groups, the rates of inhibition of cell growth of the pEGFP-MST1, 5-FU and pEGFP-MST1 + 5-FU groups were significantly increased $(\mathrm{P}<0.01)$. In addition, compared with the pEGFP-MST1 group or the 5-FU group, the inhibition rate of the pEGFP-MST1 + 5-FU group increased significantly $(\mathrm{P}<0.01)$, suggesting that the combination of MST1 and 5-FU exhibited the greatest inhibitory effect towards the proliferation of the SW480 cells.

Effect of overexpression of MST1 on colorectal cancer SW480 cell apoptosis. The results of the Hoechst 33342 staining (Fig. 3) showed that the nuclei of the control group were pale blue, and present as full circles, while the 

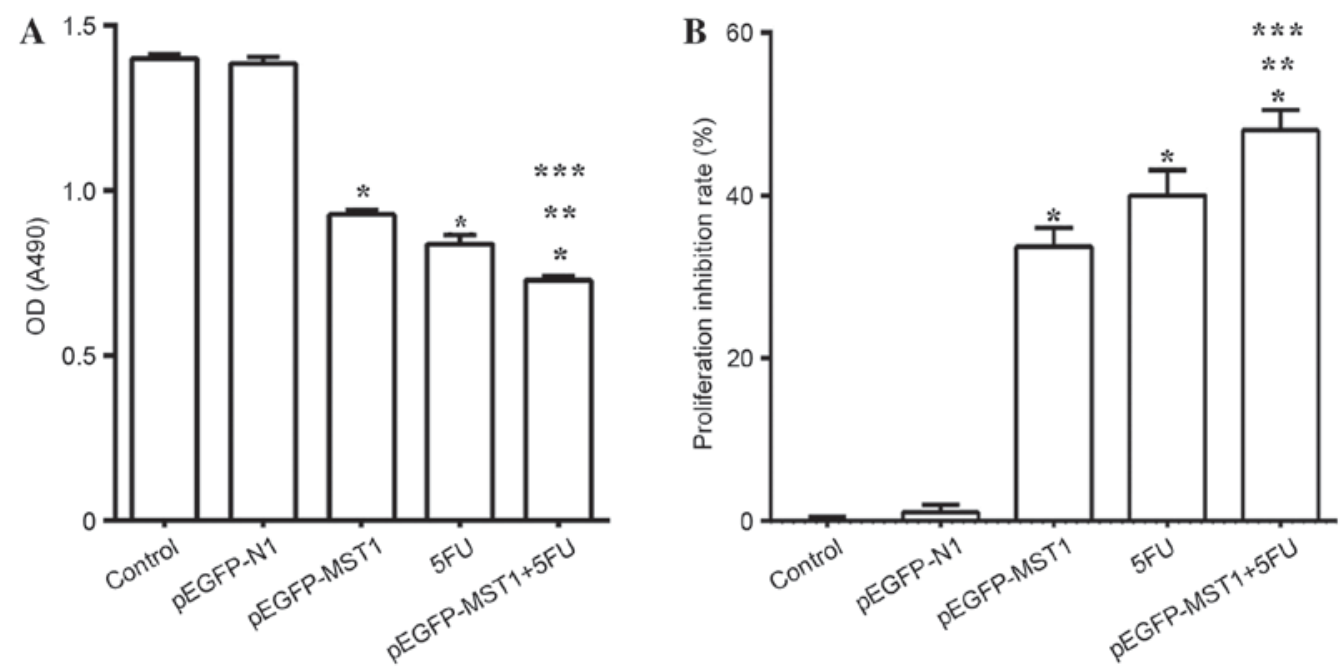

Figure 2. Cell growth of SW480 cells in each group, as detected by MTS colorimetric assay. (A) Absorbance values of SW480 cells in each group; (B) proliferation inhibition rate of SW480 cells in each group. ${ }^{*} \mathrm{P}<0.01$ vs. control or pEGFP-N1; ${ }^{* *} \mathrm{P}<0.01$ vs. pEGFP-MST1; ${ }^{* * *} \mathrm{P}<0.01$ vs. 5-FU. pEGFP, enhanced green fluorescent protein plasmid; pEGFP-N1, empty pEGFP; MST1, mammalian sterile 20-like kinase 1; 5-FU, fluorouracil; OD, optical density.
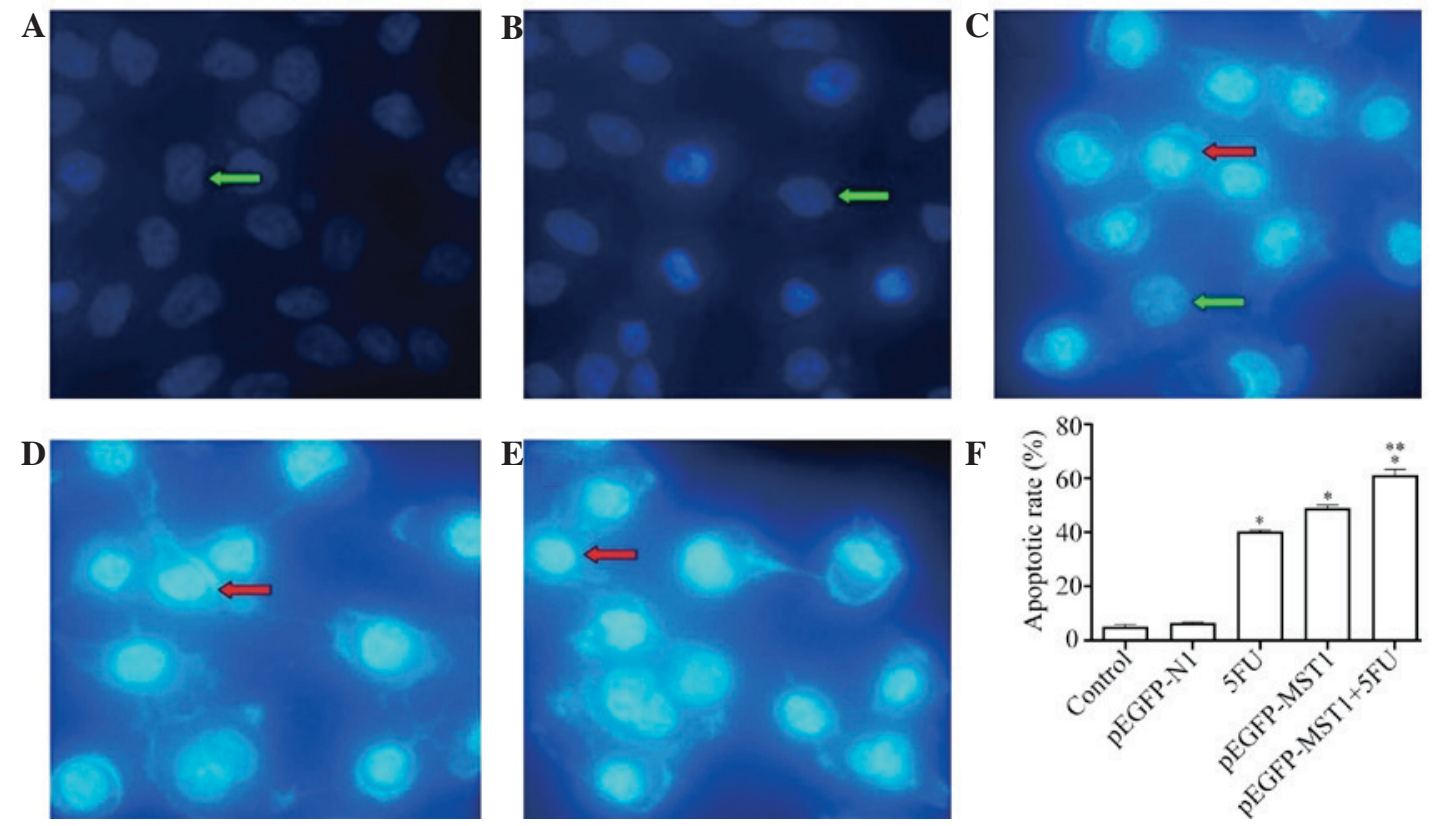

Figure 3. Hoechst 33342 staining of SW480 cells in each group (magnification, x400). (A) Control; (B) pEGFP-N1; (C) pEGFP-MST1; (D) 5-FU; (E) pEGFP-MST1 + 5-FU; (F) statistical analysis of the apoptosis rates. Darker arrows indicate the apoptotic cells and lighter arrows indicate the normal cells. ${ }^{*} \mathrm{P}<0.01$ vs. control or $\mathrm{pEGFP-N1}$; ${ }^{* *} \mathrm{P}<0.01$ vs. pEGFP-N1. pEGFP, enhanced green fluorescent protein plasmid; pEGFP-N1, empty pEGFP; MST1, mammalian sterile 20-like kinase 1; 5-FU, fluorouracil.

apoptotic nuclei were dark blue, and apoptotic bodies were observed in certain nuclei. Compared with the control and pEGFP-N1 groups, the pEGFP-MST1 group exhibited a significantly greater number of apoptotic cells $(\mathrm{P}<0.01)$. The pEGFP-MST1 + 5-FU group exhibited the largest number of apoptotic cells and, compared with the pEGFP-MST1 or the 5-FU group, the differences were statistically significant $(\mathrm{P}<0.01)$, indicating that high MST1 gene expression promoted the apoptosis of SW480 cells.

Effect of MST1 overexpression on the expression of YAP, p53, PUMA and phospho-yAP1 protein in SW480 cells. The results of the western blot analysis (Fig. 4) showed that subsequent to transfection with the pEGFP-MST1 plasmid, the level of intracellular MST1 protein increased 2.2-times more than that of the control or pEGFP-N1 groups (both $\mathrm{P}<0.01$ ), the level of phospho-YAP1 (Ser127)/YAP increased 4.5-times $(\mathrm{P}<0.01)$, and the level of $\mathrm{p} 53(\mathrm{P}<0.05)$ and PUMA proteins $(\mathrm{P}<0.01)$ also significantly increased. Compared with the control and pEGFP-N1 groups, the total level of MST of the pEGFP-MST1 + 5-FU group increased by 2.6 times (both $\mathrm{P}<0.01$ ), the level of phospho-YAP1 (Ser127)/YAP increased 17 times (both $\mathrm{P}<0.01$ ), and the levels of p53 and PUMA proteins also significantly increased (all $\mathrm{P}<0.05)$. Compared with the pEGFP-MST1 group, the total amount of MST1 in the pEGFP-MST1 + 5-FU group increased by $16.5 \%$, 

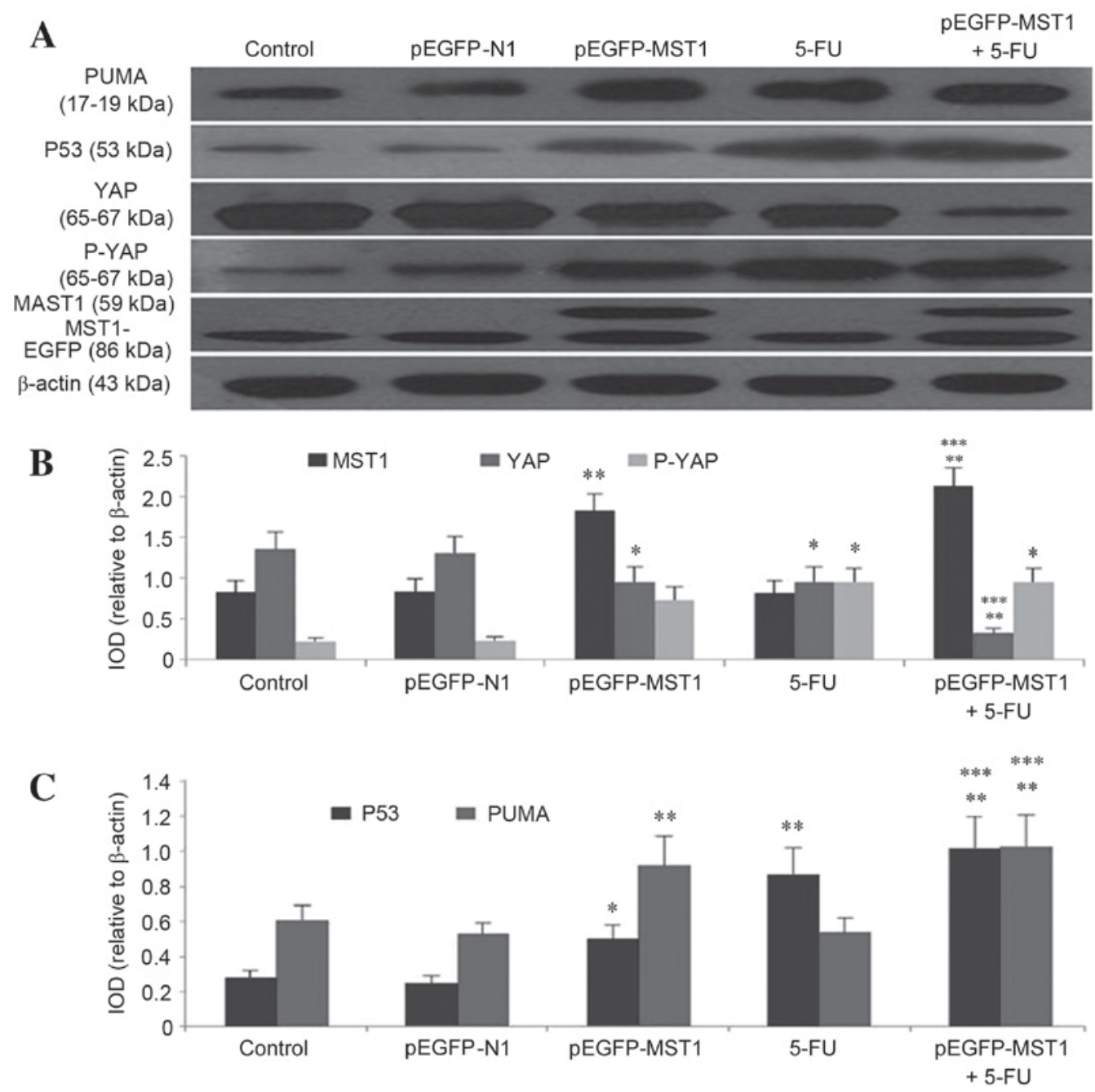

Figure 4. The combination of MST1 and 5-FU exhibited the greatest inhibitory effect on the proliferation of the SW480 cells. (A) Western blot analysis of MST1, MST1-EGFP, YAP, P-YAP1 (Ser127), p53 and PUMA of SW480 cells in each group. (B) Gray-scale value of MST1, YAP and phospho-YAP in each group. (C) Gray-scale value of P53 and PUMA. "P $<0.05$ and ${ }^{* *} \mathrm{P}<0.01$ vs. control or pEGFP-N1; ${ }^{* * *} \mathrm{P}<0.01$ vs. pEGFP-MST1 group. pEGFP, enhanced green fluorescent protein plasmid; pEGFP-N1, empty pEGFP; MST1, mammalian sterile 20-like kinase 1; 5-FU, 5-fluorouracil; PUMA, p53-upregulated modulator of apoptosis; YAP, yes-associated protein; P-YAP, phospho-yes-associated protein; IOD, integrated optical density.

phospho-YAP1 (Ser127)/YAP by $2.86 \%$ and the level of p53 and PUMA proteins also significantly increased $(\mathrm{P}<0.05)$. These results suggested that the high expression of MST1 promoted the phosphorylation of YAP protein in the SW480 cells and increased the expression levels of p53 and PUMA proteins, and that the combined application of MST1 and 5-FU exhibited a marked promotion of the phosphorylation of YAP protein in SW480 cells, as well as of the expression levels of p53 and PUMA proteins.

Effect of MST1 on the growth of human colorectal cancer SW480 cell nude mice xenografts. The results showed that the tumor inhibition rates of the pEGFP-MST1, 5-FU and pEGFP-MST1 + 5-FU groups were 48.52 $\pm 1.6,74.56 \pm 4.15$ and $87.28 \pm 2.58 \%$, respectively; compared with the control group and the negative control group, the inhibition rates of the aforementioned three groups were significantly different $(\mathrm{P}<0.01)$, and the inhibition rate of the combination treatment group was significantly higher than the pEGFP-MST1 and 5-FU groups $(\mathrm{P}<0.05)$ (Fig. 5). The results showed that exogenous MST1 exhibited a greater inhibitory effect towards human colorectal cancer nude mice xenografts, and that the combined effects of the MST1 gene + 5-FU were the greatest.

Expression levels of MST1, phospho-YAP1 (Ser127) and PUMA in nude mice xenografts. Immunohistochemistry was performed to analyze the expression of MST1, phospho-YAP1 (Ser127) and PUMA proteins in nude mice xenografts. The results showed that the positive rates of MST1 protein in the nuclei and cytoplasm of the pEGFP-MST1, 5-FU and pEGFP-MST1 + 5-FU groups increased, exhibiting a significant difference compared with the control or the empty vector treatment groups (both $\mathrm{P}<0.01$ ), although the degree of increase in the nuclei was not as high as that in the cytoplasm (Fig. 6). The positive rate of MST1 protein in the cytoplasm of the pEGFP-MST1 + 5-FU group was the highest (both $\mathrm{P}<0.01$ ). Compared with the control or the pEGFP-N1 treatment groups, the positive rate of phospho-YAP1 (Ser127) protein in the nuclei of the pEGFP-MST1, 5-FU and pEGFP-MST1 + 5-FU groups significantly increased (both $\mathrm{P}<0.01$ ) (Fig. 7). The positive rates of PUMA protein in the nuclei and cytoplasm of the 

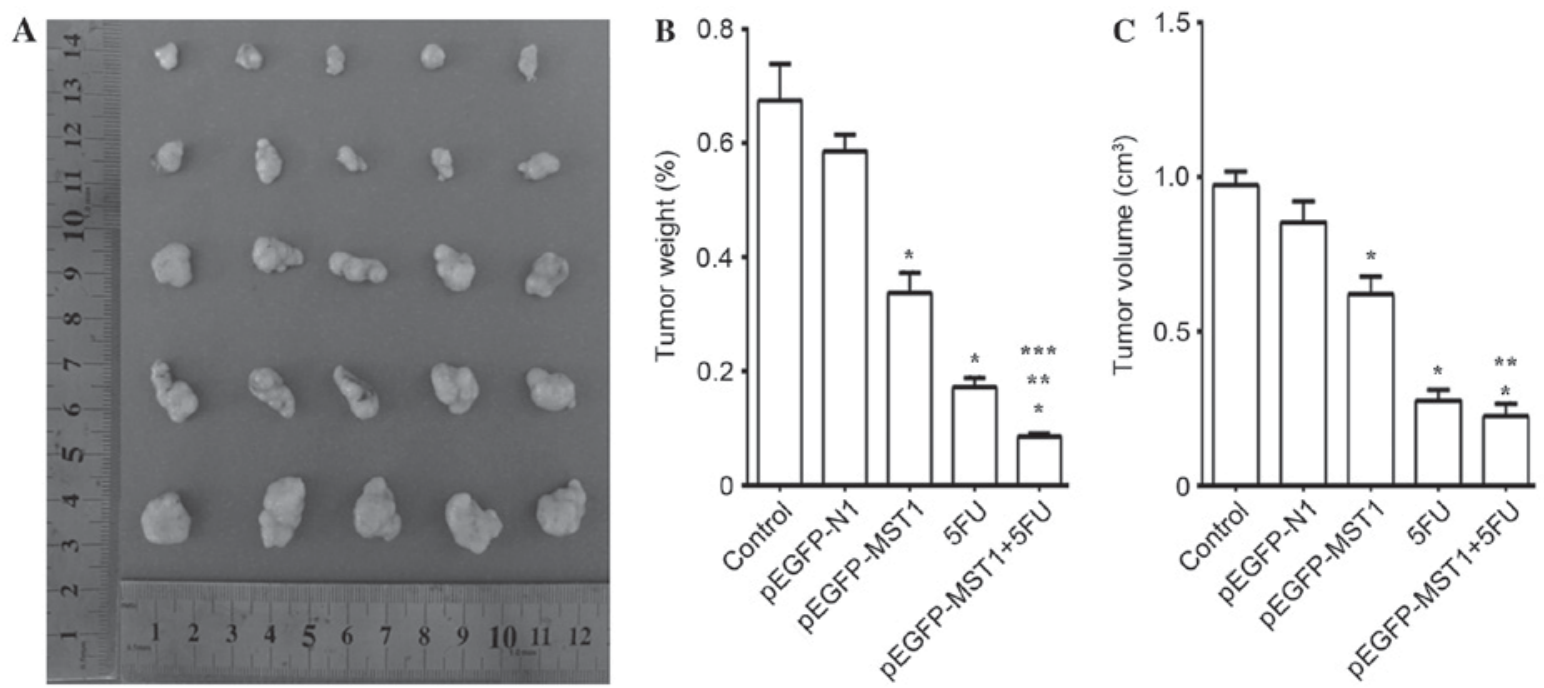

Figure 5. MST1 inhibits the growth of the human colon cancer in nude mice, while the combination treatment with MST1 and 5-FU has a greater effect. (A) Tumor xenografts from nude mice subsequent to 27 days of treatment. (B) Statistical analysis of the mean tumor weight of tumor xenografts from nude mice subsequent to 27 days of treatment. (C) Statistical analysis of the mean tumor volume of tumor xenografts from nude mice subsequent to 27 days of treatment. ${ }^{*} \mathrm{P}<0.01$ vs. control or pEGFP-N1; ${ }^{* *} \mathrm{P}<0.01$ vs. pEGFP-MST1; ${ }^{* * *} \mathrm{P}<0.05$ vs. 5 -FU. pEGFP, enhanced green fluorescent protein plasmid; $\mathrm{pEGFP-N1}$, empty pEGFP; MST1, mammalian sterile 20-like kinase 1; 5-FU, 5-fluorouracil.
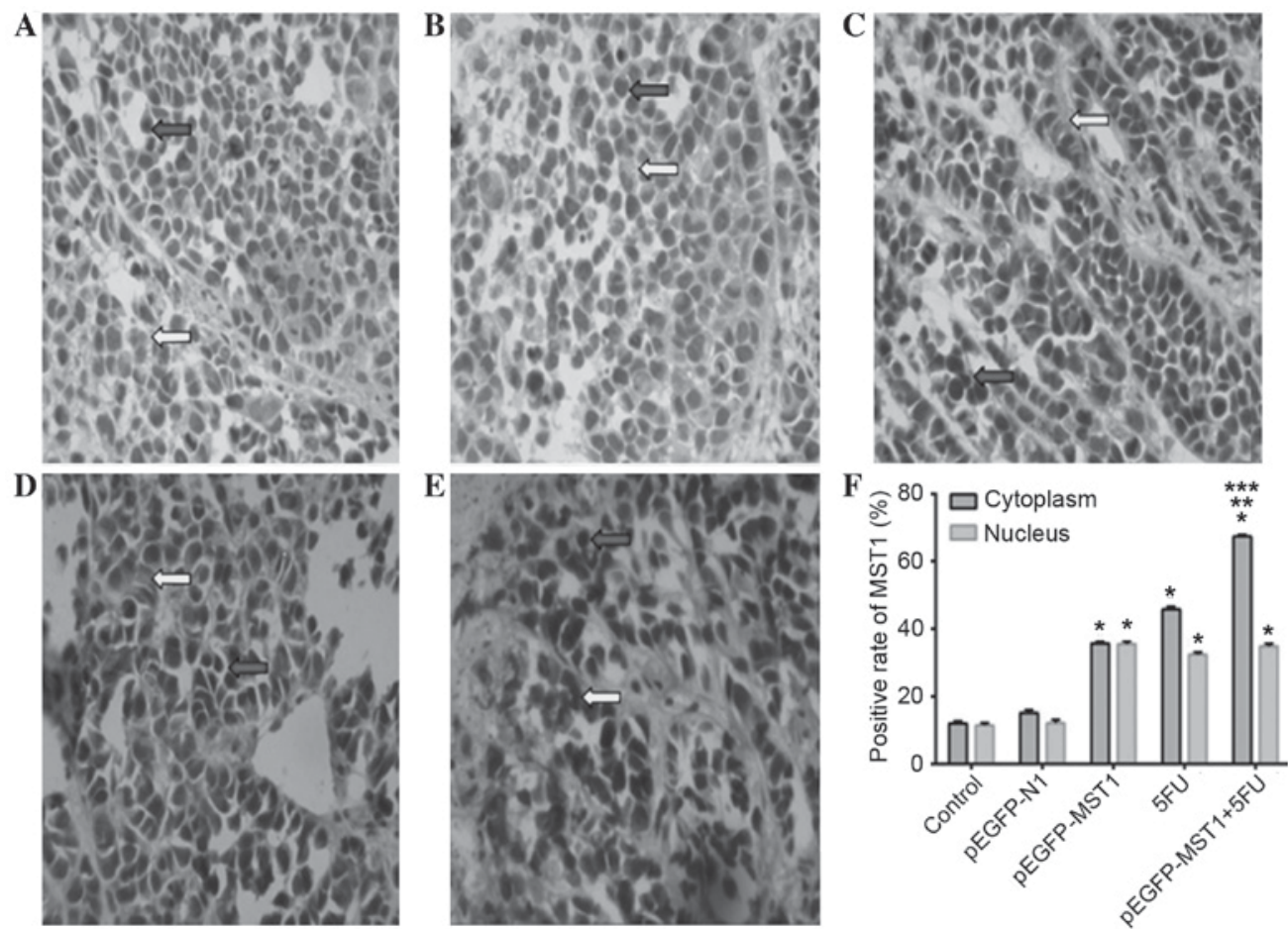

Figure 6. Immunohistochemical analysis revealed that the positive rates of MST1 protein in the pEGFP-MST1, 5-FU and pEGFP-MST1 + 5-FU groups were markedly higher than the control groups, particularly the pEGFP-MST1 + 5-FU group in cytoplasm (magnification, $\mathrm{x} 400$ ). The lighter arrows indicate cytoplasmic localization and the darker arrows indicate nuclear localization. (A) Control; (B) pEGFP-N1; (C) pEGFP-MST1; (D) 5-FU; (E) pEGFP-MST1 + 5-FU; (F) statistical analysis of MST1 expression. " $\mathrm{P}<0.01$ vs. control or pEGFP-N1; ${ }^{* *} \mathrm{P}<0.01$ vs. pEGFP-MST1; ${ }^{* * *} \mathrm{P}<0.01$ vs. 5 -FU. pEGFP, enhanced green fluorescent protein plasmid; pEGFP-N1, empty pEGFP; MST1, mammalian sterile 20-like kinase 1; 5-FU, 5-fluorouracil.

pEGFP-MST1, 5-FU and pEGFP-MST1 + 5-FU groups increased (Fig. 8), and while the degree of increase in the cytoplasm was the most significant, exhibiting significant differences compared with the control or empty vector treatment groups $(\mathrm{P}<0.01)$, the positive rate of PUMA protein in the cytoplasm of the combination treatment group was the highest $(\mathrm{P}<0.01)$.

\section{Discussion}

The MST1 protein is distributed in the cytoplasm, is highly conserved, and possesses the function of regulating the size of organs during development, cell proliferation and apoptosis, and inhibiting tumor growth (12). MST1 gene abnormalities are commonly observed in colorectal $(13,14)$, 

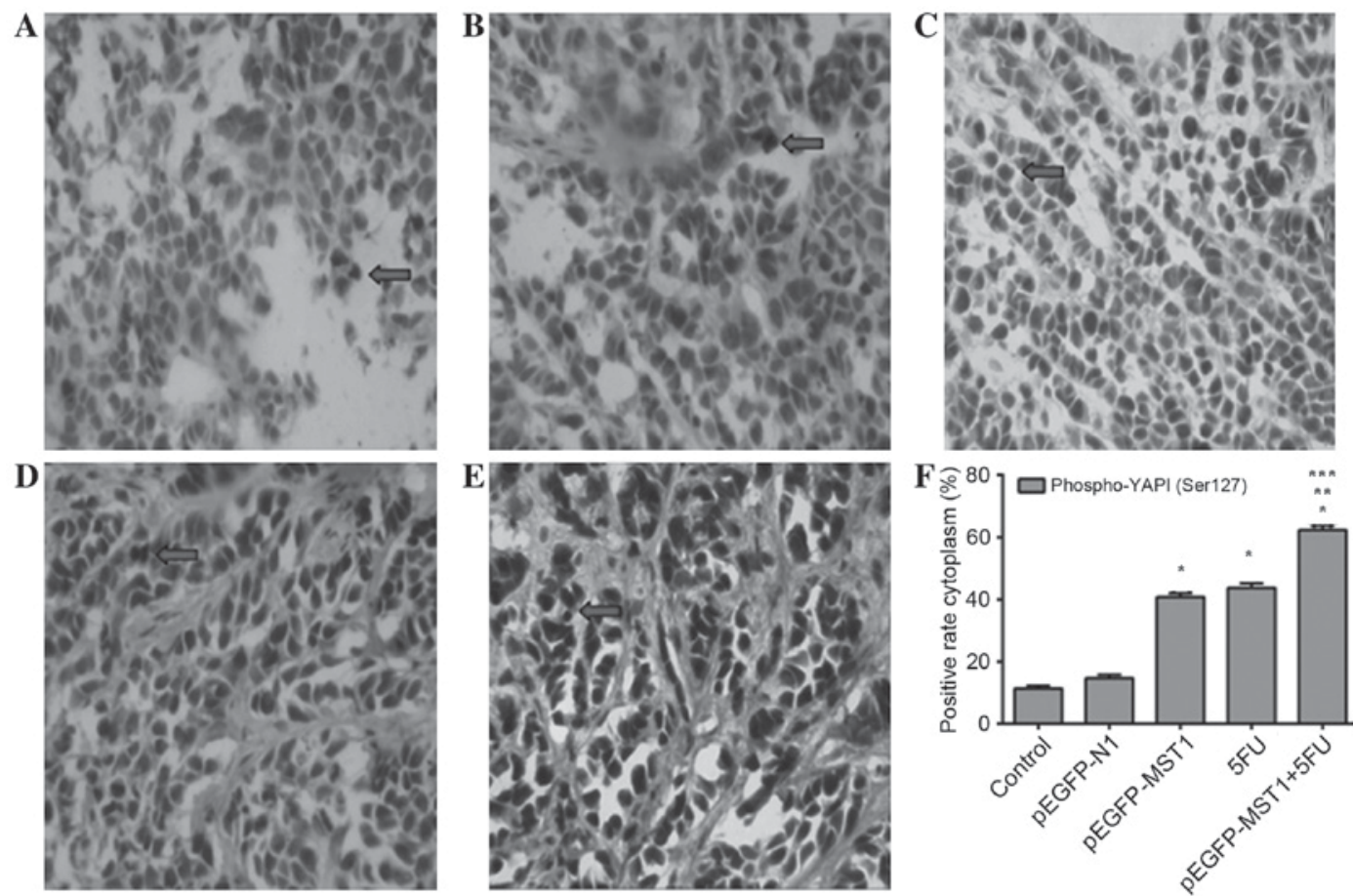

Figure 7. Immunohistochemical analysis revealed that the positive rates of phospho-YAP1 (Ser127) protein in the cytoplasm were increased significantly in the pEGFP-MST1, 5-FU and pEGFP-MST1 + 5-FU groups as compared with the control groups (magnification, $\mathrm{x} 400$ ). The arrows indicate nuclear localization (A) Control; (B) pEGFP-N1; (C) pEGFP-MST1; (D) 5-FU; (E) pEGFP-MST1 + 5-FU; (F) statistical analysis of phospho-YAP1 (Ser127) expression. "P<0.01 vs. control or, pEGFP-N1; ${ }^{* *} \mathrm{P}<0.01$ vs. pEGFP-MST1; ${ }^{* * *} \mathrm{P}<0.01$ vs. 5 -FU. pEGFP, enhanced green fluorescent protein plasmid; pEGFP-N1, empty pEGFP; MST1, mammalian sterile 20-like kinase 1; 5-FU, 5-fluorouracil; YAP, yes-associated protein.
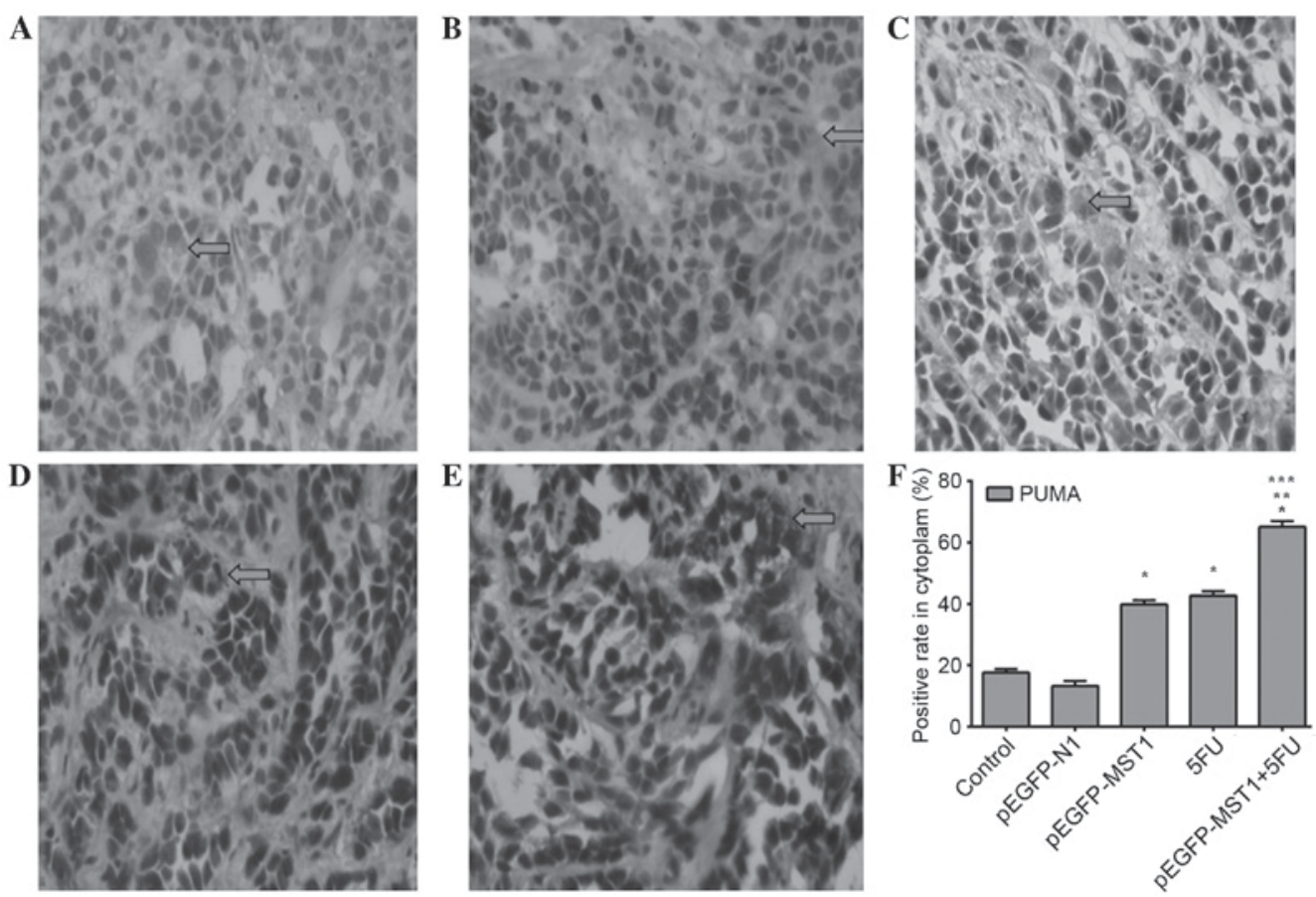

Figure 8. Immunohistochemical analysis showed that the positive rates of PUMA protein in cytoplasm were increased significantly in the pEGFP-MST1,5-FU and pEGFP-MST1 + 5-FU groups than the control groups (magnification, x400). The arrows indicate cytoplasmic localization. (A) Control; (B) pEGFP-N1; (C) pEGFP-MST1; (D) 5-FU; (E) pEGFP-MST1 + 5-FU; (F) statistical analysis of PUMA expression. ${ }^{*} \mathrm{P}<0.01$ vs. control or pEGFP-N1; ${ }^{* *} \mathrm{P}<0.01$ vs. pEGFP-MST1; ${ }^{* * * *} \mathrm{P}<0.01$ vs. 5-FU. PUMA, p53-upregulated modulator of apoptosis; pEGFP, enhanced green fluorescent protein plasmid; pEGFP-N1, empty pEGFP; MST1, mammalian sterile 20-like kinase 1; 5-FU, 5-fluorouracil.

hepatocellular $(11,15)$ and gastric $(16)$ cancer. It has also been found that the expression of YAP in the Hippo pathway is elevated in human primary colorectal cancer (17). Previous studies had found that the role of MST1 is mainly associated with the size of the liver and heart (18-20). Since then, the number of studies investigating the associations between 
MST1 and tumor cell proliferation and apoptosis has increased, and the low expression of MST1/2 (21), human Salvador 1 and Mob1 have subsequently been found in colorectal cancer (11). Additionally, it was also found that MST1-knockout mice did not inhibit the proliferation and anti-apoptosis activities of Yap1, resulting in embryonic developmental abnormality and the formation of primary hepatocellular carcinoma (10). However, one study found that liver cells lacking MST1 resisted tumor necrosis factor-induced apoptosis (16). Our previous study (11) found that the exogenously induced high expression of the MST1 gene improved MST1 kinase domain expression in HepG2 cells, upregulated the levels of Caspase-3 (CL) and phospho-YAP1 (Ser127) proteins, downregulated the levels of connective tissue growth factor, amphiregulin and survivin, and inhibited the proliferation and promoted the apoptosis of HepG2 cells. Similar results were also found in lung cancer (22). The in vitro experimental results of the present study showed that the proliferation rate of SW480 cells with MST1 overexpression significantly decreased compared with the control group (Figs. 2 and 3; $\mathrm{P}<0.01$ ), while the apoptosis rate significantly increased $(\mathrm{P}<0.01)$. The animal experimental results (Fig. 5) showed that the exogenous MST1 gene exhibited an inhibitory effect with respect to the growth of SW480 cells in nude mice, and the combined antitumor effect of the MST1 gene with 5-FU increased, indicating that MST1 inhibits the proliferation and promotes the apoptosis of human colorectal cancer SW480 cells.

Numerous studies have hypothesized that the occurrence of tumors is the co-result of multiple signaling pathways, including the Hippo signaling and p53 apoptosis pathways, possessing certain functional associations (23-26). A previous study found that in adrenal pheochromocytoma PC12 cells, YAP was phosphorylated on the no. 127 site of serine, therefore entering the nuclei and interacting with $\mathrm{p} 73$, a protein of the p53 family, resulting in apoptosis (27). In addition, p73 was revealed to activate the cytoplasmic apoptosis-associated protein PUMA in a transcription-dependent manner, thus promoting the release of cytochrome $c$ inside mitochondria, resulting in apoptosis (28). The present study found that the overexpression of MST1 promoted the phosphorylation of YAP protein in human colorectal cancer SW480 cells, and increased the expression levels of intracellular pro-apoptotic proteins, specifically p53 and PUMA. Furthermore, the combination of MST1 and 5-FU markedly promoted the phosphorylation of YAP protein and the expression of $\mathrm{p} 53$ and PUMA proteins in the SW480 cells. The immunohistochemical results in nude mice xenografts confirmed that the overexpression of MST1 promoted the phosphorylation of YAP protein, and increased the expression of p53 and PUMA proteins in human colorectal cancer SW480 cells in nude mice xenografts (Figs. 6-8), thus resulting in an increased level of apoptosis and the inhibition of the proliferation of cancer cells. This may be one of the important mechanisms of the overexpression of MST1. Therefore, the present study hypothesizes that in the occurrence and development of colorectal cancer, there may be mutual intersections with respect to the Hippo, p53 and PUMA-mediated mitochondrial apoptosis signaling pathways. As no study had investigated this previously in colorectal cancer, this theory was worthy of in-depth study.
In conclusion, the present study considered that exogenous MST1 inhibited the growth of colorectal cancer SW480 cells in nude mice xenografts, and that the mechanism of this may be via MST1 promoting the activation of cytoplasmic YAP, resulting in phospho-YAP1 (Ser127) entering the nuclei and upregulating the expression levels of p53 and PUMA, promoting the activation of caspase- 3 and inducing apoptosis. An increase in the level of MST1 may also inhibit the entry of cytoplasmic YAP into the nuclei, causing the expression levels of the pro-proliferation-associated genes to decrease, weakening the proliferation ability of the cells. Therefore, MST1 may become a novel target for the prevention and treatment of human colorectal cancer or other types of tumor.

\section{Acknowledgements}

This study was supported by the National Natural Foundation of China (grant no. 81360032).

\section{References}

1. Suo T, Mahteme H and Qin XY: Hyperthermic intraperitoneal chemotherapy for gastric andcolorectal cancer in Mainland China. World J Gastroenterol 17: 1071-1075, 2011.

2. Zhang X, Tu S, Wang Y, Xu B and Wan F: Mechanism of taurine-induced apoptosis in human colon cancer cells. Acta Biochim Biophys Sin (Shanghai) 46: 261-272, 2014.

3. Zhou GX, Li XY, Zhang Q, Zhao K, Zhang CP, Xue CH, Yang K and Tian ZB: Effects of the hippo signaling pathway in human gastric cancer. Asian Pac J Cancer Prev 14: 5199-5205, 2013.

4. Liang K, Zhou G, Zhang Q, Li J and Zhang C: Expression of Hippo pathway in colorectal cancer. Saudi J Gastroenterol 20: 188-194, 2014

5. Johnson SM, Gulhati P, Rampy BA, Han Y, Rychahou PG, Doan HQ, Weiss HL and Evers BM: Novel expression patterns of PI3K/Akt/mTOR signaling pathway components in colorectal cancer. J Am Coll Surg 210: 767-778, 2010.

6. Creasy CL and Chernoff J: Cloning and characterization of a member of the MST subfamily of Ste20-like kinases. Gene 167: 303-306, 1995.

7. Dan I, Watanabe NM and Kusumi A: The Ste20 group kinases as regulators of MAP kinase cascades. Trends Cell Biol 11: 220-230, 2001

8. Huang J, Wu S, Barrera J, Matthews K and Pan D: The Hippo signaling pathway coordinately regulates cell proliferation and apoptosis by inactivating Yorkie, the Drosophila Homolog of YAP. Cell 122: 421-434, 2005.

9. Dong J, Feldmann G, Huang J, Wu S, Zhang N, Comerford SA, Gayyed MF, Anders RA, Maitra A and Pan D: Elucidation of a universal size-control mechanism in Drosophila and mammals. Cell 130: 1120-1133, 2007.

10. Zhou D, Conrad C, Xia F, Park JS, Payer B, Yin Y, Lauwers GY, Thasler W, Lee JT, Avruch J and Bardeesy N: Mst1 and Mst2 maintain hepatocyte quiescence and suppress hepatocellular carcinoma development through inactivation of the Yap1 oncogene. Cancer Cell 16: 425-438, 2009.

11. Xu C, Liu C, Huang W, Tu S and Wan F: Effect of Mst1 overexpression on the growth of human hepatocellular carcinoma HepG2 cells and the sensitivity to cisplatin in vitro. Acta Biochim Biophys Sin (Shanghai) 45: 268-279, 2013.

12. Ling P, Lu TJ, Yuan CJ and Lai MD: Biosignaling of mammalian Ste20-related kinases. Cell Signal 20: 1237-1247, 2008.

13. Zhou D, Zhang Y, Wu H, Barry E, Yin Y, Lawrence E, Dawson D, Willis JE, Markowitz SD, Camargo FD and Avruch J: Mst1 and Mst2 protein kinases restrain intestinal stem cell proliferation and colonic tumorigenesis by inhibition of Yes-associated protein (Yap) overabundance. Proc Natl Acad Sci USA 108: E1312-E1320, 2011.

14. Xu ZP, Zhu JS, Zhang Q and Wang XY: A breakdown of the Hippo pathway in gastric cancer. Hepatogastroenterology 58: 1611-1617, 2011. 
15. Konsavage WM Jr, Kyler SL, Rennoll SA, Jin G and Yochum GS: $\mathrm{Wnt} / \beta$-catenin signaling regulates Yes-associated protein (YAP) gene expression in colorectal carcinoma cells. J Biol Chem 287: 11730-11739, 2012.

16. Song H, Mak KK, Topol L, Yun K, Hu J, Garrett L, Chen Y, Park O, Chang J, Simpson RM, et al: Mammalian Mst1 and Mst2 kinases play essential roles in organ size control and tumor suppression. Proc Natl Acad Sci USA 107: 1431-1436, 2010.

17. Cai J, Zhang N, Zheng Y, de Wilde RF, Maitra A and Pan D: The Hippo signaling pathway restricts the oncogenic potential of an intestinal regeneration program. Genes Dev 24: 2383-2388, 2010.

18. Avruch J, Zhou D, Fitamant J and Bardeesy N: MST1/2 signalling to Yap: Gatekeeper for liver size and tumour development Br J Cancer 104: 24-32, 2011

19. Odashima M, Usui S, Takagi H, Hong C, Liu J, Yokota M and Sadoshima J: Inhibition of endogenous MST1 prevents apoptosis and cardiac dysfunction without affecting cardiac hypertrophy after myocardial infarction. Circ Res 100: 1344-1352, 2007.

20. Yamamoto S, Yang G, Zablocki D, Liu J, Hong C, Kim SJ Soler S, Odashima M, Thaisz J, Yehia G, et al: Activation of MST1 causes dilated cardiomyopathy by stimulating apoptosis without compensatory ventricular myocyte hypertrophy. J Clin Invest 111: 1463-1474, 2003.

21. Minoo P, Zlobec I, Baker K, Tornillo L, Terracciano L, Jass JR and Lugli A: Prognostic significance of mammalian sterile20-like kinase 1 in colorectal cancer. Mod Pathol 20: 331-338, 2007.
22. Xu CM, Liu WW, Liu CJ, Wen C, Lu HF and Wan FS: Mst1 overexpression inhibited the growth of human non-small cell lung cancer in vitro and in vivo. Cancer Gene Ther 20: 453-460, 2013.

23. Strano S, Fausti F, Di Agostino S, Sudol M and Blandino G: PML surfs into HIPPO tumor suppressor pathway. Front Oncol 3: 36, 2013.

24. Ardestani A, Paroni F, Azizi Z, Kaur S, Khobragade V, Yuan T, Frogne T, Tao W, Oberholzer J, Pattou F, et al: MST1 is a key regulator of beta cell apoptosis and dysfunction in diabetes. Nat Med 20: 385-397, 2014.

25. Chiang J and Martinez-Agosto JA: Effects of mTOR inhibitors on components of the Salvador-Warts-Hipoo pathway. Cell 1: 886-904, 2012.

26. Maejima Y, Kyoi S, Zhai P, Liu T, Li H, Ivessa A, Sciarretta S, Del Re DP, Zablocki DK, Hsu CP, et al: Mstl inhibits autophagy by promoting the interaction between Beclin 1 and Bcl-2. Nat Med 19: 1478-1488, 2013

27. Zhang H, Wu SN and Xing D: Inhibition of A $\beta(25-35)$-induced cell apoptosis by low-power-laser-irradiation (LPLI) through promoting Akt-dependent YAP cytoplasmic translocation. Cell Signal 24: 224-232, 2012.

28. Yoon MK, Ha JH, Lee MS and Chi SW: Structure and apoptotic function of p73. BMB Rep 48: 81-90, 2015. 